\title{
Formalization and Verification of Dubbo Using CSP
}

\author{
Zhiru Hou, Jiaqi Yin, Huibiao Zhu* \\ Shanghai Key Laboratory of Trustworthy Computing, \\ East China Normal University, Shanghai, China
}

\begin{abstract}
Dubbo is a high-performance, lightweight Java Remote Procedure Call (RPC) framework developed by Alibaba, which provides interface-oriented remote method call, intelligent fault tolerance and automatic service registration. Since Dubbo is extensively applied recently as an excellent representative RPC framework, it is of great significance to formally analyze Dubbo. In this paper, we use Communicating Sequential Processes (CSP) to model and formalize Dubbo. In order to enhance the reliability of the call, we use token authentication mechanism in the modeling process. Moreover, we put the CSP description of the established model into the model checker Process Analysis Toolkit (PAT) for simulation and verification. We verify whether the four properties are valid, including Deadlock Freedom, Connectivity, Robustness and Parallelism. Our final verification results show that the model can satisfy these properties, thus we can conclude the framework can guarantee the highly available remote call.

Index Terms-Dubbo, Formalization, Verification, CSP
\end{abstract}

\section{INTRODUCTION}

With the development of the Internet, the architecture for a large number of website applications is constantly changing, from Monolithic Architecture, Vertical Architecture, Distributed Service Architecture to Flow Computing Architecture. Now, more and more website technicians choose to use Microservices [1], which is evolved from ServiceOriented Architecture (SOA) [3]. As a means of communication, Remote Procedure Call (RPC) [2] still plays an important role in Microservices, and Apache Dubbo is an excellent representative of the RPC framework.

Dubbo [4] is an open source and high-performance RPC call framework developed by Alibaba. It is a RPC remote call service solution dedicated to providing high performance and transparency. In recent years, some work has been done on Dubbo [5, 6]. Zhang et al. [5] proposed a distribution network state control system using Dubbo in order to improve the lean management level of the distribution network. Xiong et al. [6] designed a new type of think tank evaluation system based on Microservices, and realized the communication between services based on the RPC remote call of Dubbo distributed framework. From these works, we can find that they focused more on using Dubbo to implement remote calls between services. Unfortunately, there is nearly no research conducted to describe the interactions in Dubbo formally, thus it is a challenge to give a formal model on the interactions between the components in Dubbo.

In this paper, we propose a formal model of Dubbo using Communicating Sequential Processes (CSP) [7], which aims to reflect the interactions of Dubbo's call process. In order

\footnotetext{
${ }^{*}$ Corresponding author: hbzhu@sei.ecnu.edu.cn (H. Zhu).
}

to better ensure the reliability of calling services, token authorization mechanism is also formalized in this model. In addition, we use Process Analysis Toolkit (PAT) [8, 11] to verify whether the achieved model caters for some significant properties or not, including Deadlock Freedom, Connectivity, Robustness and Parallelism.

The remainder of this paper is organized as follows. Section II gives a brief introduction to Dubbo and the process algebra CSP. In Section III, we formalize the model of Dubbo using CSP. Furthermore, in Section IV, we apply the model checker PAT to implement the achieved model and verify four properties. Finally, we give a conclusion and make a discussion on the future work in Section V.

\section{BACKGROUND}

In this section, we give a brief introduction to Dubbo's call service process, token authentication and process algebra CSP.

\section{A. Dubbo}

Dubbo is a distributed service framework. The architecture of Dubbo is shown in Fig. 1. As we have seen in Fig. 1, Dubbo architecture mainly has four components, including provider, consumer, registry and monitor. Furthermore, Fig. 1 shows the main communications of Dubbo architecture, and their respective functionalities are seen in Table I.

TABLE I

Components And Functionalities of DubBo

\begin{tabular}{ll}
\hline Components & Functionalities \\
\hline Provider & Exposing remote services \\
Consumer & Calling the remote services \\
Registry & $\begin{array}{l}\text { Service discovery and configuration } \\
\text { Monitor }\end{array}$ \\
& $\begin{array}{l}\text { Counting the number of service invoca- } \\
\text { tions and time-consuming }\end{array}$ \\
Container & Managing the services' lifetime \\
\hline
\end{tabular}

In Fig. 1, when consumer wants to call the service it needs, the following sequence of actions occurs:

(1) Container is responsible for launching, loading and running the provider.

(2) Provider registers its services to registry when it starts.

(3) Consumer subscribes the needed services from the registry when it starts.

(4) Registry returns a list of providers to consumer. When the list changes, the registry will push the changed data to consumer through long connection. 


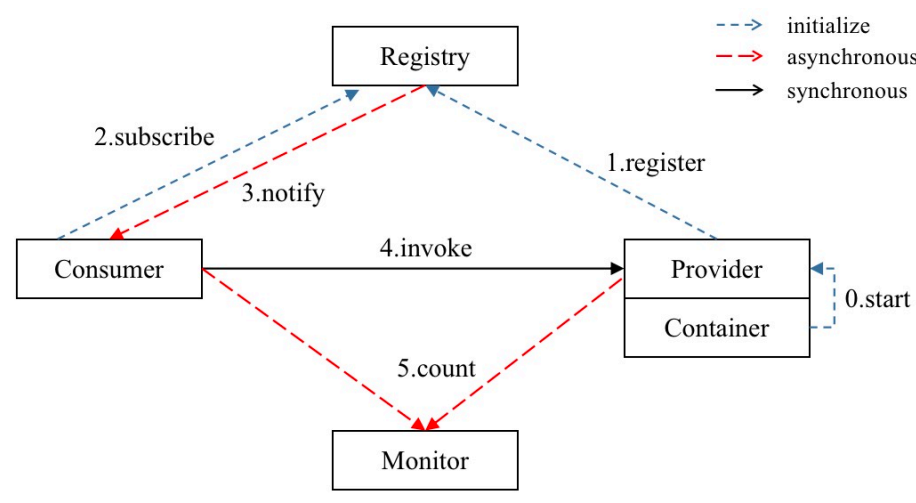

Fig. 1. Communications of Dubbo Module (Adapted from [4])

(5) Consumer selects one of the providers based on load balancing algorithm and executes the invocation. If fails, it will choose another provider.

(6) When monitor starts, it will subscribe all providers and consumers that registered or called.

(7) Both consumer and provider count the number of service invocations and time-consuming in memory, and send the statistics to monitor every minute.

In Dubbo, if the provider wants to verify the identity of the consumer before the consumer invokes its service, the system can use token authentication between them. In this condition, the consumer cannot bypass the registry and connect directly to provider. The details of using token authentication in Dubbo can be seen in Fig. 2.

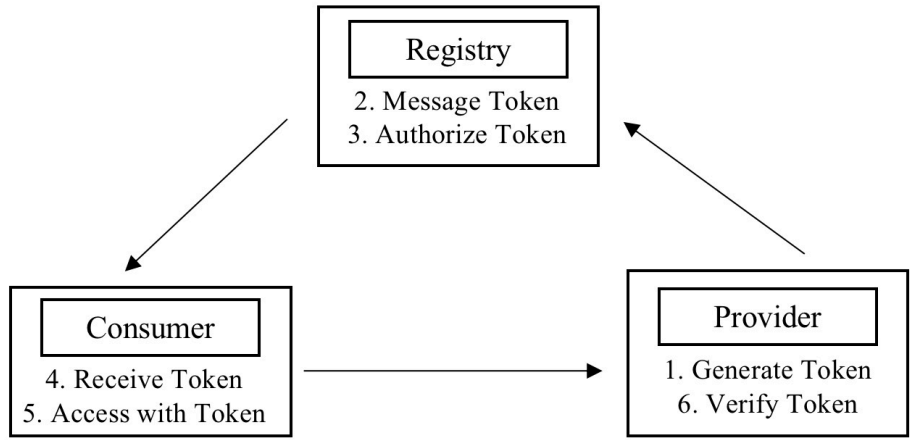

Fig. 2. Token Authentication of Dubbo (Adapted from [4])

There are two types of tokens in Dubbo, which are random token and fixed token. Random token is generated using a UUID, and fixed token is equivalent to the password which is used in this paper. The sequence of using token verification [12] in Dubbo is:

(1) When provider registers its service, it generates a token and publishes it with the service to registry.

(2) Registry has the right to decide whether to assign token to consumer.

(3) Once the consumer obtains the URL of the provider from registry, it can request to invoke the provider through the token.
(4) The provider needs to verify whether this token is consistent with the token generated by itself. If it is nonconsistent, this invocation will fail.

\section{B. A Brief Introduction to CSP}

CSP was proposed by C.A.R Hoare, which is the abbreviation of Communicating Sequential Processes [7]. It has been successfully applied to model and verify diverse concurrent systems and protocols $[9,10]$. We use the following syntax to define the processes in this paper.

$$
\begin{aligned}
P, Q::= & S K I P|S T O P| a \rightarrow P|c ? x \rightarrow P| c ! e \rightarrow P \mid \\
& P \triangleleft b \triangleright Q|P \square Q| P \| Q|P||Q| P ; Q
\end{aligned}
$$

- $S K I P$ stands for a process which terminates successfully.

- $S T O P$ represents that the process does nothing and runs into the deadlock state.

- $a \rightarrow P$ performs action $a$ firstly, then behaves like $P$.

- $c ? x \rightarrow P$ receives a message by channel $c$ and assigns the received message to variable $x$, then behaves like $P$ subsequently.

- $c$ ! $e \rightarrow P$ sends a message $e$ through channel $c$, then the subsequent behavior is $P$.

- $P \triangleleft b \triangleright Q$ represents a conditional choice. If the expression $b$ is true, process $P$ will be carried out; otherwise, process $Q$ is executed.

- $P \square Q$ is a general choice, it acts like either $P$ or $Q$ and the environment decides the selection.

- $P \| Q$ shows the parallel composition between $P$ and $Q$. The $\|$ means that actions in the alphabet of both operands require simultaneous participation of them.

- $P\|\| Q$ indicates that $P$ interleaves $Q$ which means $P$ and $Q$ run concurrently without barrier synchronization.

- $P ; Q$ executes process $P$ and process $Q$ in sequence.

\section{Modeling DubBo}

In this section, we give a formal model of Dubbo's call service process, and this model includes five components. The formalization proceeds based on the four components described in Section II. In order to better describe the temporal process of Dubbo, we propose a new component Clock.

\section{A. Overall Modeling}

For the whole system, there are four crucial processes running in parallel through their own corresponding channels, including Provider, Consumer, Registry and Clock. Monitor process interleaves with them. The behavior of Dubbo system process is modelled as below.

DubSys ${ }_{d f}$ Provider $\|$ Consumer $\|$ Registry $\|$ Clock; System $={ }_{d f}$ DubSys $\| \mid$ Monitor;

Next, we give the formalization of Provider, Consumer, Registry, Monitor and Clock, respectively. 
TABLE II

The Explanations Of Channels Of The Model

\begin{tabular}{ll}
\hline Channels & Functionalities \\
\hline$P_{i} R$ & $\begin{array}{l}\text { Transmitting register messages between } \\
\text { providers and registry } \\
C_{d} R\end{array}$ \\
$\begin{array}{l}\text { Transmitting subscribe messages between } \\
\text { consumers and registry } \\
P_{i} C_{d}\end{array}$ & $\begin{array}{l}\text { Transmitting call messages between con- } \\
\text { sumers and providers }\end{array}$ \\
$C_{d} M$ & $\begin{array}{l}\text { Transmitting consumers' monitor mes- } \\
\text { sages between consumers and monitor }\end{array}$ \\
$P_{i} M$ & $\begin{array}{l}\text { Transmitting providers' monitor messages } \\
\text { between providers and monitor }\end{array}$ \\
ComHeart & $\begin{array}{l}\text { Transmitting heartbeat messages between } \\
\text { providers and registry } \\
\text { Transmitting time messages }\end{array}$ \\
&
\end{tabular}

\section{B. Provider}

In this system, there can be several providers. Each provider has a unique ID marked as $i$, and $I$ is the total number about providers. Provider is mainly responsible for providing services and generating tokens. In addition, Provider periodically sends a heartbeat to registry and a monitor message to monitor. Thus, we formalize Provider as below.

Provider $=_{d f}\|\|_{i \in I}$ Service $_{i}$

Service $_{i}=_{d f}$ ServProvider $\|||$ ServPMon $\| \mid$ ServHBeat

Before introducing the three processes of Provider, we first explain messages and channels used here. The messages can be described as follows, and the explanations of channels are illustrated in Table II.

- ProInfo is sent from Provider to Registry, which contains the ID, IP address, host name and the corresponding information of the Provider.

- InvokeSuccess is a reply from Provider to Consumer, which means that Consumer can call the matched Provider successfully.

- InvokeFail is a reply from Provider to Consumer, which represents that the invocation fails.

- TokenFail is a reply from Provider to Consumer, which means that the token sent by Consumer and the token of Provider are inconsistent.

- MonPro is transmitted from Provider to Monitor, which owns the ID, the number of service invocations and time-consuming of the Provider.

- request is used by asking Clock the current time.

- HeartBeat is sent from Provider to Registry, which indicates the Provider is still running.

- ProListInfo is transferred from Provider to Monitor, which contains the URL addresses of all providers.

ServProvider. ServProvider describes the details of publishing services and being called by consumers. At first, Provider registers its services and token to Registry. When
Provider receives the call request from Consumer, it first verifies whether the token provided by consumer matches the token generated by itself. If the match is successful, the authentication is passed; otherwise, the authentication fails and TokenFail is sent to Consumer. In addition, when the monitor starts, Provider sends ProListInfo to Monitor asynchronously. The behavior of ServProvider is modelled as below.

$$
\begin{aligned}
& \text { ServProvider }={ }_{d f}
\end{aligned}
$$

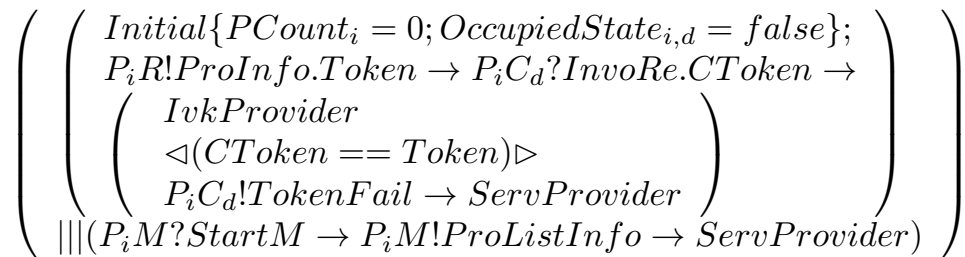

For the process IvkProvider, since the authentication passes, it is necessary to check whether the provider is occupied by other services. If the provider is not occupied, Provider sends InvokeSuccess to Consumer. It also increases the number of service invocations and calculates timeconsuming using the process Clock; otherwise, the consumer can wait timeout seconds. Suppose consumer can call the provider within timeout seconds, the call is successful; otherwise it fails. The detailed behavior is modelled as follows.

$$
\begin{aligned}
& \text { IvkProvider }={ }_{d f}
\end{aligned}
$$

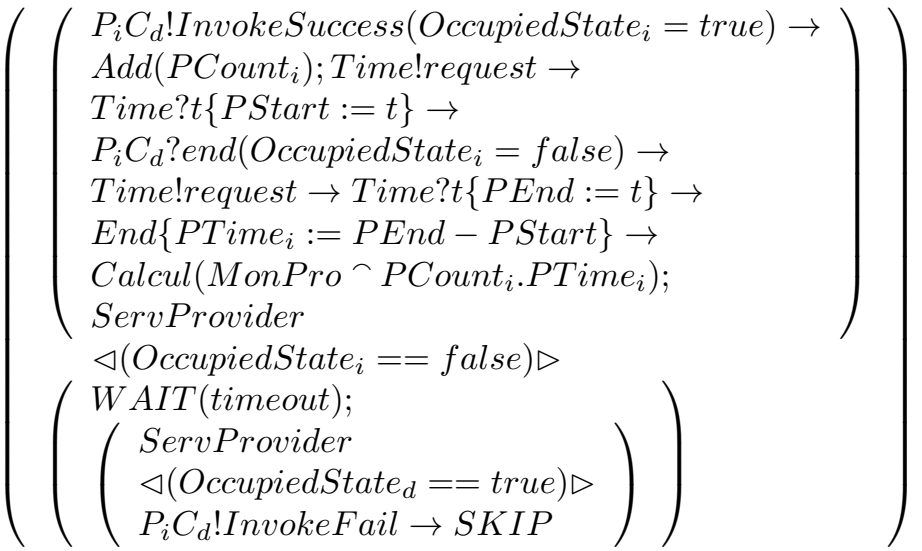

The following is the model of the $W A I T$ function, where the parameter $t$ is the unit of time to wait, and the specific model is as follows.

$$
W A I T(t)={ }_{d f} S K I P \triangleleft(t==0) \triangleright(t i c k \rightarrow W A I T(t-1))
$$

ServPMon. ServPMon process is mainly used to send monitor messages to monitor regularly. Once the monitor starts, it asks the current time and waits MonInterval seconds. Then it sends MonPro to Monitor and cycles continuously. Next we give the formalization of ServPMon.

$$
\begin{aligned}
\text { ServPMon }= & d f \text { Time!request } \rightarrow \text { Time } ? \text { start } \rightarrow \\
& \text { W AIT }(\text { MonInterval }) ; P_{i} \text { M!MonPro } \rightarrow \\
& \text { ServPMon }
\end{aligned}
$$


ServHBeat. ServHBeat works in heartbeat mechanism, which means that Provider needs to send a heartbeat to Registry regularly. Then we formalize the process of ServHBeat as below.

$$
\begin{aligned}
& \text { ServHBeat }=_{d f} \text { Time!request } \rightarrow \text { Time } ? \text { start } \rightarrow \\
& \left(\begin{array}{c}
\left(\begin{array}{c}
\text { ComHeart } i \text { HeartBeat } \rightarrow \\
\text { Assign }(\text { last }:=\text { start }) ; \text { ServHBeat }
\end{array}\right) \\
\triangleleft(\text { start }- \text { last }>\text { HBeatInterval }) \triangleright \\
\text { ServHBeat }
\end{array}\right)
\end{aligned}
$$

Provider asks Clock for the current time firstly. If the time interval is less than HBeatInterval, Provider sends a request to Clock again; otherwise, Provider sends HeartBeat to Registry directly and this process cycles continuously.

\section{Consumer}

Like Provider, each consumer has a unique ID marked as $d$, and $D$ is the total number about consumers. Consumer mainly expresses subscribing service and calling service. Moreover, Consumer sends a monitor message to monitor regularly. Thus, we formalize Consumer as below.

Consumer $=_{d f}\|\|_{d \in D}$ Subscriber ${ }_{d}$

Subscriber $_{d}={ }_{d f}$ ServConsumer $\|||$ ServCMon

The messages in Consumer can be described as follows.

- SusRe is sent from Consumer to Registry, which contains the ID, IP address and the corresponding information of the Consumer.

- InvoRe is transmitted from Consumer to Provider, including the IDs of Consumer and Provider together with invocation request.

- MonCon is sent from Consumer to Monitor, which contains the ID, the number of service invocations and time-consuming of the Consumer.

- end is transmitted from Consumer to Provider, which means that Consumer wants to finish the call process.

- ConListInfo is transferred from Consumer to Monitor, which owns the URL addresses of all consumers.

ServConsumer. ServConsumer focuses more on subscribing services and initiating the call processes. After Consumer sends subscription to Registry, Consumer can attain a list of providers and the tokens from Registry. Then Consumer verifies whether the states of providers are available or not. Consumer can select an available provider to call via load balancing algorithm. Moreover we use Random Load Balance algorithm here, which is selected according to the provider's weight and sets a random probability. Consumer sends invocation request to Provider and waits the reply. In addition, if ProList changes, Registry will notify Consumer asynchronously. Once monitor starts,
Consumer needs to send ConListInfo to Monitor. After the above analysis, ServConsumer is formalized as below.

$$
\begin{aligned}
& \text { ServConsumer }=_{d f} \\
& \left.\qquad \begin{array}{l}
\left(\begin{array}{l}
C_{d} R ! \text { SusRe } \rightarrow C_{d} R ? \text { ProList } \rightarrow \\
\left(\begin{array}{l}
\text { IvkConsumer } \\
\triangleleft\left(\text { state }_{i}==\text { open }\right) \triangleright \text { SKIP }
\end{array}\right)
\end{array}\right) \\
\|\left(C_{d} R ? \text { ModiProList } \rightarrow \text { SKIP }\right) \\
\|\left(C_{d} M ? \text { Start } M \rightarrow C_{d} M ! \text { ConListInfo } \rightarrow\right. \\
\text { ServConsumer })
\end{array}\right)
\end{aligned}
$$

For IvkConsumer, if reply is InvokeSuccess, it calculates time and increases the number of invocations as Provider; by contrast, it can have two opportunities to try to call other providers. Then, we formalize the process IvkConsumer as below.

$$
\begin{aligned}
& \text { IvkConsumer }={ }_{d f}
\end{aligned}
$$

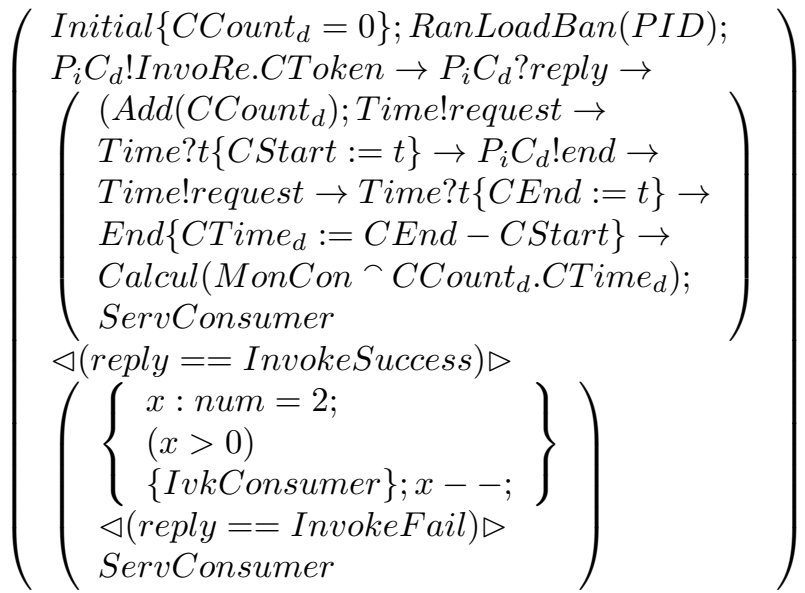

ServCMon. ServCMon process is mainly used by consumer to send monitor messages to monitor regularly. Once the monitor starts, Provider needs to send MonCon to Monitor. Like ServPMon, we give the formalization of ServCMon.

$$
\begin{aligned}
\text { ServCMon }= & d f \text { Time } ! \text { request } \rightarrow \text { Time } ? \text { start } \rightarrow \overrightarrow{ } \\
& \text { WAIT }(\text { MonInterval }) ; C_{d} \text { M!MonCon } \rightarrow \\
& \text { ServCMon }
\end{aligned}
$$

\section{Registry}

We use Zookeeper [13] to implement dynamic registration and discovery of services in the registry. Registry serves as a component for storing information and receiving the heartbeat message from providers. Thus, we formalize Registry as below.

\section{Registry ${ }_{d f}$ ServRegistry $\|$ RegHBeat}

The messages in Registry can be described as follows, and the channels are explained in Table II.

- ProList is sent from Registry to Consumer, and it is a list which contains matching providers' information. 
- ModiProList is transferred from Registry to Consumer, which owns modified matching providers' information.

Next, we formalize the two processes, respectively.

ServRegistry. ServRegistry process is applied for describing the registration and subscription processes. Firstly Registry receives registration from Provider and subscription from Consumer, respectively. Based on the information provided by Consumer, Registry checks whether there is a matching provider. If there is no matching provider, then it skips; otherwise, Registry finds out the relevant providers according to the matching algorithm SelectPro, and sends ProList to Consumer. The behavior of ServRegistry process is modelled as below.

$$
\begin{gathered}
\text { ServRegistry }=_{d f} \text { Initial }\{\text { ProList }=\text { null }\} ; \\
P_{i} \text { R?ProInfo.Token } \rightarrow C_{d} \text { R?SusRe } \rightarrow \\
\left(\begin{array}{c}
\text { SelectPro(ProList } \frown \text { ProInfo.IP.Token }) ; \\
C_{d} R ! \text { ProList } \rightarrow \text { ServRegistry } \\
\triangleleft(\text { SusRe.CInfo } \in \text { ProInfo.PSer }) \triangleright \text { SKIP }
\end{array}\right)
\end{gathered}
$$

RegHBeat. $R e g H B$ eat process mainly involves the heartbeat mechanism. The process RegHBeat is formalized as follows.

$$
\begin{aligned}
& \text { RegHBeat }{ }_{d f}
\end{aligned}
$$

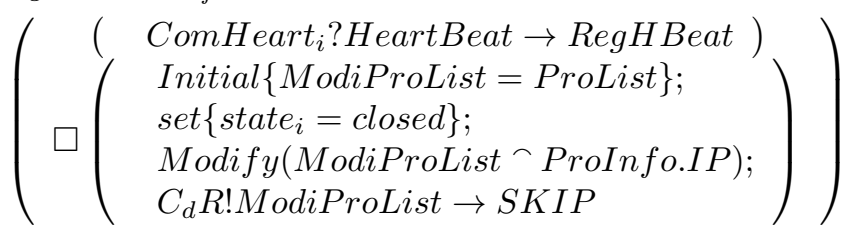

In case Registry receives heartbeat message from Provider, it indicates the provider is running normally; on the other hand, it means that the provider may be down, and we can modify the provider's information to ModiProList.

\section{E. Monitor}

Monitor is responsible for monitoring the status of the service. Thus, Monitor can be formalized as below.

$$
\begin{aligned}
\text { Monitor }= & d f \text { P } P_{i} M ! \text { Start } M \rightarrow P_{i} \text { M?ProListInfo } \rightarrow \\
& C_{d} M ! \text { Start } M \rightarrow C_{d} M \text { ? ConListInfo } \rightarrow \\
& P_{i} M ? \text { MonPon } \rightarrow C_{d} M ? \text { MonCon } \rightarrow \\
& \text { Monitor }
\end{aligned}
$$

When monitor starts, it needs to obtain the URL information of all providers and consumers. It also receives MonPro from Provider and MonCon from Consumer, respectively.

\section{F. Clock}

In order to better represent the temporal process of Dubbo, we abstract Clock process, which is used to express the global clock. Once other processes ask Clock for the time via the channel Time, Clock will send back the current time $t$ which is a positive integer. The processes of $C l o c k(t)$ can be described as follows.

$$
\begin{aligned}
\text { Clock }(t)={ }_{d f} & (\text { tick } \rightarrow \text { Clock }(t+1)) \\
& \square(\text { Time? request } \rightarrow \text { Time! } \rightarrow \text { Clock }(t))
\end{aligned}
$$

\section{VERIFICATION}

In this section, we implement CSP model mentioned in Section III and verify some important properties using PAT.

\section{A. Verification in PAT}

Before verifying the properties, we define some significant variables. $I, D, R, M$ denote the number of the providers, the consumers, the registry and the monitor. In the trial, we set $I$, $D, R, M$ to be $2,3,1,1$, respectively.

\section{Property 1: Deadlock Freedom}

In Dubbo, we should avoid the situation that two or more consumers are waiting the resources which have been occupied by other consumers infinitely. In addition, System 1() should also meet Deadlock Freedom. For the explanation of System 1(), see Property 2. In the tool PAT, there is a primitive to describe this situation:

$$
\begin{aligned}
& \text { \#assert System() deadlockfree; } \\
& \text { \#assert System } 1(\text { ) deadlockfree; }
\end{aligned}
$$

\section{Property 2: Connectivity}

Registry and monitor are optional, and consumer can connect provider directly in Dubbo. However, we use token to enhance identity authentication in this paper, so that consumers need to go through registry to connect with the provider. Thus we prove that monitor is optional here.

We hide the relevant channels of monitor to detect whether the provider can successfully connect with consumer without monitor, we use System1() to model this in PAT. If the monitor is optimal, the variable CncStatePro and CncStateCon should be True. Moreover, both System() and System 1() should satisfy this property. The assertion about this property is defined as below:

$$
\begin{gathered}
\text { System } 1()=\text { System }() \backslash\left\{P_{i} M, C_{d} M\right\} ; \\
\text { \#define Connectivity }(\text { CncStatePro }==\text { true \&\& } \\
\text { CncStateCon }==\text { true }) ; \\
\text { \#assert System }() \text { reaches Connectivity; } \\
\text { \#assert System } 1() \text { reaches Connectivity; }
\end{gathered}
$$

\section{Property 3: Robustness}

The primary objective of Dubbo is to accomplish the call of provider reliably even in the presence of failures. If providers are stateless, one instance's downtime does not affect the usage. After all the providers of one service go down, consumer infinitely reconnects to wait for service provider to recover.

In this paper, we assume that the services called by consumers are the same as those provided by providers. Here we define that there are four valid conditions listed as follows. The first and second conditions are that all providers can run normally, the third condition is that the first provider is down 
and the last condition is that the second provider is down. The assertion is defined as below:

$$
\begin{aligned}
& \text { \#define Robust1(PCount }[0]==1 \& \& \\
& P \text { Count }[1]==2) \text {; } \\
& \text { \#define Robust2(PCount }[0]==2 \& \& \\
& P \text { Count }[1]==1) \text {; } \\
& \text { \#define Robust3(PCount }[0]==0 \& \& \\
& \text { PCount }[1]==3) \text {; } \\
& \text { \#define Robust4(PCount }[0]==3 \& \& \\
& \text { PCount }[1]==0) \text {; } \\
& \text { \#define Robustness(Robust1||Robust2 } \\
& \text { ||Robust3||Robust4); } \\
& \text { \#assert System() reaches Robustness; }
\end{aligned}
$$

\section{Property 4: Parallelism}

Parallelism means that the system allows multiple providers publish services and consumers subscribe services concurrently, the processes do not interfere with each other. We define two Boolean variables, aplPro means the number of registration submissions of providers, and aplCon means the number of subscription submissions of consumers. Our goal is that the system can reach a state where the value of aplCon and aplPro should be 1 , which reflects the providers and the consumers can involve calling processes parallelly. The assertion about this property is defined as below:

$$
\begin{gathered}
\text { \#define Para } 1(\text { aplPro }[0]==1 \& \& \text { aplPro }[1]==1) \\
\text { \#define Para } 2(\text { aplCon }[0]==1 \& \& \text { aplCon }[1]==1 \\
\text { \&\& aplCon }[2]==1) ; \\
\text { \#define Parallelism }(\text { Para } 1 \text { \&\& Para } 2) ; \\
\text { \#assert System }() \text { reaches Parallelism; }
\end{gathered}
$$

\section{B. Verification Results}

The verification results are showed in Fig. 3. From Fig. 3 , we can easily find that the four properties are all valid, which represents that the constructed model caters for the specifications and these properties.

1) Deadlock Freedom means that the constructed model

\begin{tabular}{|c|c|}
\hline Asserti & \\
\hline Q 1 & System0 deadlockfree \\
\hline (1) 2 & System 10 deadlockfree \\
\hline ( 3 & System 0 reaches Connectivity \\
\hline (1) 4 & System 10 reaches Connectivity \\
\hline (2) 5 & System 0 reaches Robustness \\
\hline (Q) 6 & System0 reaches Parallelism \\
\hline
\end{tabular}
does not run into a deadlock state.

2) Connectivity is valid which means that the provider and the consumer can connect successfully, even without monitor.

3) Robustness represents that the framework has good fault tolerances, which is an important property for RPC framework.

4) Parallelism indicates that the providers can commit registrations and the consumers can commit subscriptions concurrently.

\section{CONCLUSiOn AND Future Work}

Dubbo is a high-performance distributed service framework from Alibaba, which can provide good remote call. In this paper we analyzed Dubbo and used token mechanism to
Verification - Dubbo.csp

Fig. 3. Verification Results

enhance identity authentication. We applied process algebra CSP in formalizing Dubbo. Subsequently, we used PAT to encode the CSP description and verified this model. In addition, we performed the validation of four properties, including Deadlock Freedom, Connectivity, Robustness and Parallelism. These properties are all valid. Therefore, we can conclude that our model satisfies these properties and the framework can realize effective remote calls from the perspective of process algebra.

The formal verification of the distributed service framework is still a challenge. In the future, we will formalize and verify the Dubbo with Zookeeper [13] in more details and verify whether the framework can resist attacks or not.

Acknowledgements. This work was partly supported by National Key Research and Development Program of China (Grant No. 2018YFB2101300), National Natural Science Foundation of China (Grant No. 61872145, 62032024), Shanghai Collaborative Innovation Center of Trustworthy Software for Internet of Things (Grant No. ZF1213).

\section{REFERENCES}

[1] Microservices, [Online] Available: https://martinfowler.com/articles /microservices.html.

[2] A. D. Birrell and B. J. Nelson, "Implementing remote procedure calls", ACM Transactions on Computer Systems, vol. 2, no. 1, pp. 39-59, 1984.

[3] N. M. Josuttis, SOA in Practice: The Art of Distributed System Design. O'Reilly, 2007.

[4] Dubbo. [Online]. Available: http://dubbo.apache.org.

[5] Y. Zhang, Y. Liu, B. Li and L. Li, "Research on Distribution Network Status Management System Based on Cloud Platform", 2019 International Joint Conference on Information, Media and Engineering, pp. 391-395, 2019.

[6] S. Xiong and B. Huang, "A Novel Think Tanks Evaluation System Based on Micro Service", in Journal of Physics: Conference Series, 2021.

[7] C. A. R. Hoare, Communicating sequential processes. Prentice Hall International in Computer Science, 1985.

[8] PAT: Process analysis toolkit, [Online] Available: http://pat.comp. nus.edu.sg/.

[9] G. Lowe and B. Roscoe, "Using CSP to Detect Errors in the TMN protocol", IEEE Transactions on Software Engineering, vol. 25, no .10, pp. 659-669, 1997.

[10] A. W. Roscoe and J. Huang, "Checking noninterference in timed CSP", Formal Aspects of Computing, vol. 25, no. 1, pp. 3-35, 2013.

[11] J. Sun, Y. Liu and J. S. Dong, "Model checking CSP revisited: Introducing a process analysis toolkit", International Symposium On Leveraging Applications of Formal Methods, Verification and Validation, pp. 307-322, 2008.

[12] J. Yi and L. Lin, Deep understanding of Apache Dubbo and actual combat. House of Electronics Industry, 2019.

[13] Zookeeper. [Online]. Available: https://zookeeper.apache.org. 